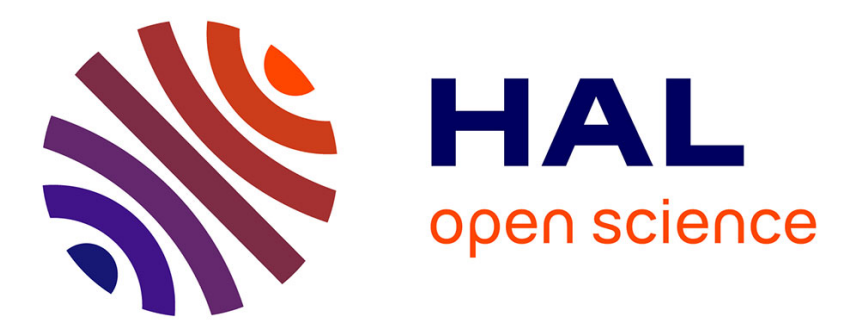

\title{
Sono-activated ultrasound localization microscopy
}

Yann Desailly, Olivier Couture, Mathias Fink, Mickaël Tanter

\section{To cite this version:}

Yann Desailly, Olivier Couture, Mathias Fink, Mickaël Tanter. Sono-activated ultrasound localization microscopy. Applied Physics Letters, 2013, 103 (17), pp.174107. 10.1063/1.4826597 . hal-02481847

\section{HAL Id: hal-02481847 \\ https://hal.science/hal-02481847}

Submitted on 17 Feb 2020

HAL is a multi-disciplinary open access archive for the deposit and dissemination of scientific research documents, whether they are published or not. The documents may come from teaching and research institutions in France or abroad, or from public or private research centers.
L'archive ouverte pluridisciplinaire HAL, est destinée au dépôt et à la diffusion de documents scientifiques de niveau recherche, publiés ou non, émanant des établissements d'enseignement et de recherche français ou étrangers, des laboratoires publics ou privés. 


\section{Sono-activated ultrasound localization microscopy}

Yann Desailly, Olivier Couture, Mathias Fink, and Mickael Tanter

Citation: Appl. Phys. Lett. 103, 174107 (2013);

View online: https://doi.org/10.1063/1.4826597

View Table of Contents: http://aip.scitation.org/toc/apl/103/17

Published by the American Institute of Physics

\section{Articles you may be interested in}

Subwavelength far-field ultrasound drug-delivery

Applied Physics Letters 109, 194102 (2016); 10.1063/1.4967009

Ultrasound resolution beats the diffraction limit

Physics Today 69, 14 (2016); 10.1063/PT.3.3069

A model for large amplitude oscillations of coated bubbles accounting for buckling and rupture

The Journal of the Acoustical Society of America 118, 3499 (2005); 10.1121/1.2109427

Ultrafast ultrasound localization microscopy

The Journal of the Acoustical Society of America 141, 3951 (2017); 10.1121/1.4988974

Resolution beyond the diffraction limit in the acoustic microscope: A nonlinear effect Journal of Applied Physics 56, 1338 (1984); 10.1063/1.334124

Wideband acoustic activation and detection of droplet vaporization events using a capacitive micromachined ultrasonic transducer

The Journal of the Acoustical Society of America 139, 3193 (2016); 10.1121/1.4953580

\section{Scilight}

Sharp, quick summaries illuminating the latest physics research

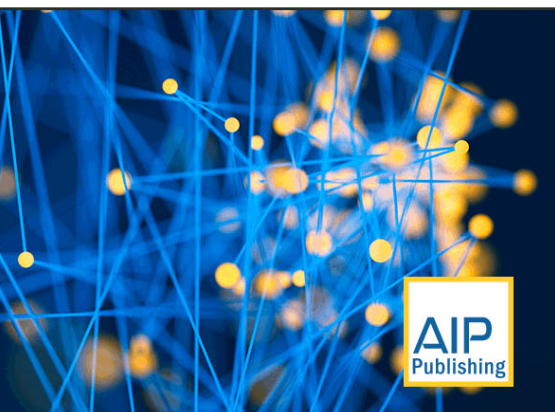




\title{
Sono-activated ultrasound localization microscopy
}

\author{
Yann Desailly, Olivier Couture, ${ }^{\text {a) }}$ Mathias Fink, and Mickael Tanter \\ Institut Langevin (ESPCI, CNRS, INSERM), 1 rue Jussieu, 75005 Paris, France
}

(Received 19 August 2013; accepted 8 October 2013; published online 25 October 2013)

\begin{abstract}
Scanned at very high ultrasound frame rates, injectable microbubbles can be activated sequentially as isolated punctual sources of acoustic echoes. These signals can thus be localized far beyond the diffraction limit. The resolution improvement granted by Sono-Activated Ultrasound Localization Microscopy (SAULM) was demonstrated within microfluidic channels 20 times smaller than the imaging wavelength $(\lambda=870 \mu \mathrm{m})$. The width of the channels mapped with SAULM was 13 times smaller than as they appeared under conventional ultrasound imaging. Two channels separated by $\lambda / 4.5$ could be distinguished. Implementing SAULM in-vivo could lead to a complete reconstruction of the vascular tree down to the smallest capillaries at several centimeter depth. @ 2013 AIP Publishing LLC. [http://dx.doi.org/10.1063/1.4826597]
\end{abstract}

The resolution of an imaging system is intrinsically linked to the propagation regime of the wave scanning the medium. Indeed, three different regimes describe wave propagation through tissues: coherent, diffusive, and near-field regimes leading to completely different resolutions. In conventional optical microscopy, the optical wave keeps its coherence during propagation and the optical wavelength controls the spatial resolution of the final image. Thus, according to diffraction theory, the resolution of optical microscopy is limited at best to a half-wavelength. For instance, biological structures separated by less than $300 \mathrm{~nm}$ become indistinguishable. To image smaller cellular organelles, for example, either the shorter wavelengths of electrons in the coherent regime or near-field effects (for which the resolution is no more linked to the wavelength but rather to the observation distance) can be exploited. ${ }^{1}$

Recently, in the coherent propagation regime, localization microscopy (generalized terminology for several techniques referred to as Fluorescence Photo-activation Localization Microscopy (fPALM), Stochastic Optical Reconstruction Microscopy (STORM), etc.) has been shown to surpass the diffraction limit. ${ }^{2-4}$ This technique exploits random distributions of fluorescent sources which are too close to be resolved but can be activated, one by one, to form a series of images where the emitters are isolated in time (punctual) and well separated. Since their images do not interfere, the actual position of the source can be found from the summit of the individual point-spread function. This localization of a point-source is not submitted to Rayleigh criterion and can be performed with a resolution much smaller than the wavelength. The fluorescent sources being stochastically activated or inactivated between each acquisition, a map of their positions can be reconstructed after a series of acquisitions. Although time-consuming, these blinking and bleaching techniques can distinguish details at a level of $50 \mathrm{~nm}$ at $650 \mathrm{~nm}$ wavelength $(\lambda / 13)$. When photoactivated sources are bound to specific biological structures, they can highlight nanometric structures such as podosomes, tubulin, and talin strands. ${ }^{5}$ Introduced by Hess et al. in 1996,

a)e-mail: olivier.couture@espci.fr this concept of localization microscopy revolutionized the field of optical imaging in the last decade.

In biomedical imaging, ultrasonic waves rely on their ability to propagate tens of centimeters without losing their coherence. Since that distance is typically orders of magnitude greater than the wavelength of $0.1-1 \mathrm{~mm}$, waves propagate in the coherent regime and the spatial resolution in ultrasound depends on the wavelength. Again, due to diffraction theory, the resolution is limited at best to a half-wavelength $(\sim 0.1-1 \mathrm{~mm})$ and details below $100 \mu \mathrm{m}$ cannot be distinguished at clinical frequencies $(8 \mathrm{MHz})$. Shorter wavelength can be used, but to the cost of a higher attenuation and lower penetration, which rapidly reduces the scope of the imaging technique. ${ }^{6}$ To date, this "resolution versus penetration" paradigm has confounded the quest for ultrasound microscopy deep into tissues.

However, similar to fluorescence microscopy, ultrasound can also rely on very efficient contrast agents at the cellular level. Indeed, radiologists can inject a solution of microbubbles intravenously to improve the blood contrast or molecular targets in ultrasound images. ${ }^{7}$ Because of their gaseous content and their resonance frequency within clinical range, these agents, $1-5 \mu \mathrm{m}$ in size, scatter sound very efficiently. Moreover, because of their high compressibility, they behave in a nonlinear fashion which permits the use of several microbubble-specific imaging techniques. ${ }^{8}$ Hence, Ultrasound Contrast Imaging can map the vascular network with a much higher sensitivity than conventional ultrasound. Of course, clouds of microbubbles randomly circulating within the vascularization cannot be resolved by ultrasound and contrast imaging is also limited to the resolution of ultrasound imaging ( $100 \mu \mathrm{m}$ to $1 \mathrm{~mm})$.

Microbubbles are highly sensitive contrast agents, and it is possible to image a single microbubble deep into the human body with acoustical wavelength several orders of magnitude longer than the diameter of the microbubble. Such a high sensitivity could already allow a form of ultrasound localization. Indeed, if individual bubbles were injected at low concentrations and, hence, separated by several wavelengths, their position could be retrieved at resolution surpassing diffraction. But, such an approach would be very unsatisfactory due to the time required to reconstruct a complete microvasculature with very few moving microbubbles. 
To attain comparable results than FPALM in ultrasound, a method that distinguishes individual bubbles within a concentrated cloud is necessary.

Beyond their resonance and nonlinearity, microbubbles can also be pushed or destroyed by a sufficiently large ultrasound pulse transmission ${ }^{9}$ and undergo drastic changes in their scattering characteristics within milliseconds, as shown by ultrafast optical and acoustical imaging. ${ }^{10,11}$ This phenomenon is stochastic since each microbubble, being of different size and surrounded by a different environment, should respond to ultrasound differently. At appropriate transmit acoustical pressure, it could thus be possible to activate and detect bubbles one by one. Consequently, microbubbles could act as acoustically activated sources, which could then be exploited for ultrasound localization microscopy beyond the diffraction limit leading to sono-activated ultrasound localization microscopy (SAULM), an acoustic analog of FPALM optical microscopy. However, to perform SAULM the frame rate required to observe these fast events cannot be attained by conventional focused ultrasound (frame rates $<100 \mathrm{~Hz}$ ) as it becomes here mandatory to detect transient events at the millisecond scale over large regions of interest.

Frame rates up to $20000 \mathrm{~Hz}$ have already been introduced using ultrafast ultrasound scanner in the context of supersonic shear wave imaging, ${ }^{12,13}$ micro-doppler, ${ }^{14}$ and functional imaging. ${ }^{15}$ In ultrafast imaging, a single ultrasound plane wave is used to illuminate the whole sample, and the resulting echoes are collected with multiple parallel elements (Figure 1(b)). The radio-frequency signal from each channel is then reconstructed in the computer to create an image. Within the timescale of a single pulse-echo (around $50 \mu \mathrm{s}$ ), a complete map of the tissue observed is obtained rather than a single line in conventional imaging (Figure 1(a)). With ultrafast imaging, high acquisition rates (beyond $1000 \mathrm{~Hz}$ ) become reachable which enables the observation of fast events within the sample, such as the disruption and motion of the bubbles.

The current study is an experimental demonstration of the concept of sono-activated ultrasound localization
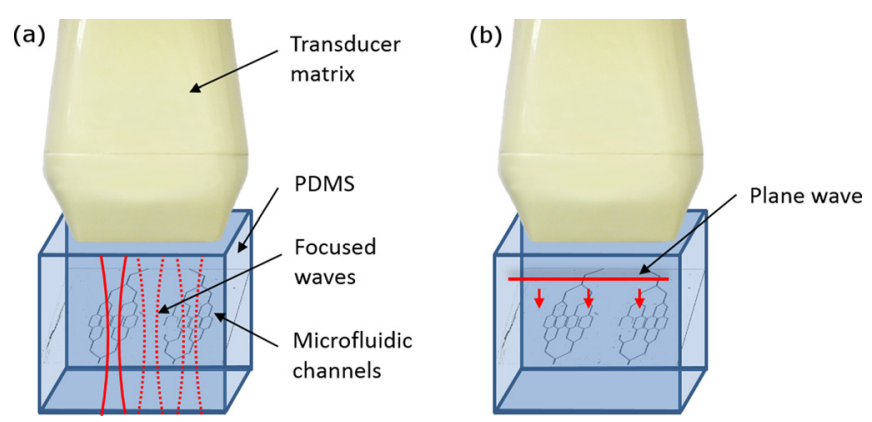

FIG. 1. Plane wave imaging compared to focused imaging. These two techniques were applied on microchannels inside PDMS imaged with a 1.75 MHz 2D transducer matrix connected to an ultrafast ultrasonic scanner (ultrafast frame rate: $1 \mathrm{kHz}$ ). (a) Conventional focused imaging is performed by scanning a focused beam over each line of an image which limits the frame rate to about $100 \mathrm{~Hz}$. (b) In ultrafast plane wave imaging, a single unfocused wave is emitted through the sample and echoes from individual scatterers are acquired by multiple reception channels. The radiofrequency (RF) signal of each of these channels is then beamformed, resulting in frame rates up to $20 \mathrm{kHz}$. microscopy within an in-vitro model of the vasculature. Plane wave ultrafast imaging was implemented on an ultrasound clinical scanner with 128 fully programmable emission-reception channels (Aixplorer, Supersonic Imagine, France) at a rate of one thousand frames per second. To demonstrate sono-activated ultrasound localization microscopy, a low frequency $(1.75 \mathrm{MHz})$ transducer was used with a resolution limited by diffraction to 1040 micrometers. For such experiments, it is also necessary to access all of the three spatial coordinates, adding the requirement for a two-dimensional ultrasonic matrix rather than a conventional linear array. However, as SAULM is not bounded by the same rules as diffraction-limited imaging, the transducer matrix can be undersampled in space. In theory, the localization of the bubble in the elevation axis can indeed be performed as soon as two independent elements are available along the elevation axis. The localization along this axis is indeed obtained from independent travel path measurements, i.e., two independent arrival times measured with a typical $0.1 \mu$ s precision. Thus, 2 parallel series of 64 transducers (lateral pitch: $0.4 \mathrm{~mm}$, elevation pitch: $1.5 \mathrm{~mm}$ ) were placed over the sample to be imaged and connected to the ultrasound scanner. This setup was first used to implement conventional ultrasound imaging, which consists in imposing delays on each electronic channel to sequentially focus the ultrasonic beam on each line of the image.

To compare conventional focused imaging and sonoactivated ultrasound localization microscopy, their respective resolutions were tested by imaging bubbles flowing through microchannels printed by lithography in polydimethylsiloxane (PDMS). The channels were between 40 and 100 microns in width and $80 \mu \mathrm{m}$ in depth. The microfluidic system was designed to simulate the vasculature network in order to avoid acoustic impedance discontinuities. It was tilted in space, to simulate a 3D network. In the center, the channels were separated by 50 to $200 \mu \mathrm{m}$. Two channels from the same parent channel are separated by one wavelength so that they could not be distinguished by any standard ultrasound imaging method at the selected transmit frequency.

Sulfur hexafluoride bubbles $(1-3 \mu \mathrm{m}$ in size-SonoVue by Bracco Research SA) at a dilution between $10^{4}$ and $10^{5}$ per milliliter were pushed into the channels at a constant flow rate by a syringe pump $((200 \mathrm{~mL} / \mathrm{min})$ Harvard Apparatus). This microbubbles concentration corresponds typically to 10 to 100 bubbles located within the region of interest at each acquisition. With such concentration, the wave fronts backscattered by this cloud of bubbles cannot be resolved by conventional ultrasound (see Fig. 2(a)). Each of the acquisitions consisted in 50000 ultrafast images. Three datasets were acquired with three different positions for the transducer matrix in order to cover the whole microfluidic system (see Fig. 3(d)).

Sono-activated ultrasound localization microscopy was implemented by emitting plane waves at an acoustic pressure of $450 \mathrm{kPa}$ (peak-negative pressure at $4 \mathrm{~cm}$ depth) with a frame rate of $1000 \mathrm{~Hz}$ toward the bubble-filled vascular phantom. The acquired data were the backscattered echoes coming from all the scatterers present within the whole sample. The radiofrequency signals acquired from successive transmissions 
(a)

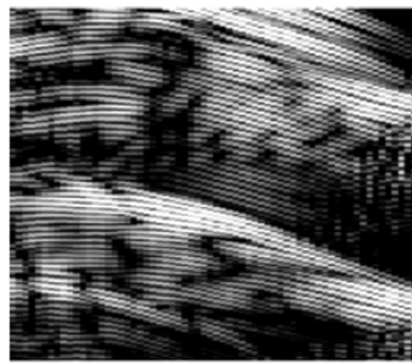

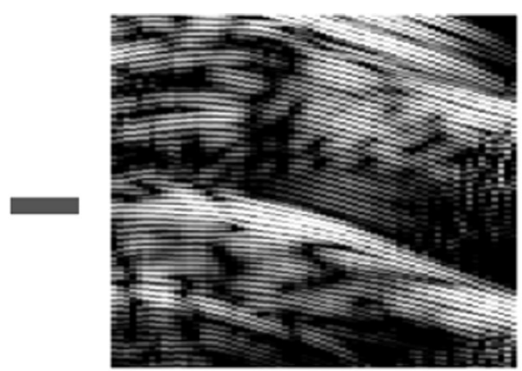

(c)
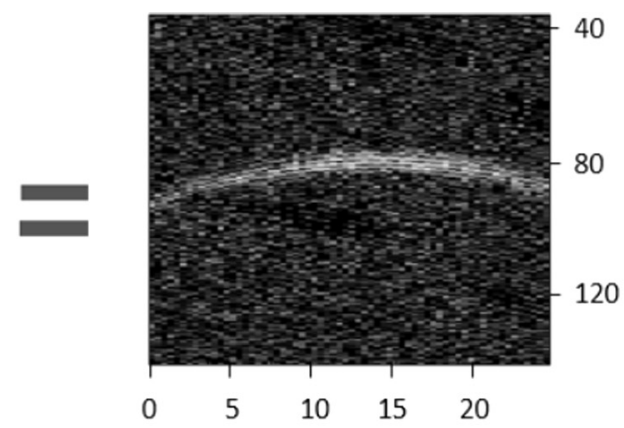

Lateral position ( $\mathrm{mm}$ ) (b)

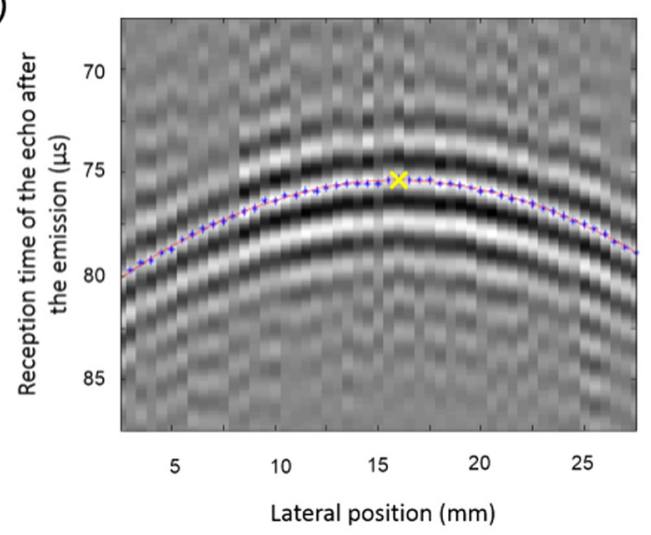

* Regression point $\quad \begin{gathered}\text { Resulting summit } \\ \text { of the parabola }\end{gathered}$

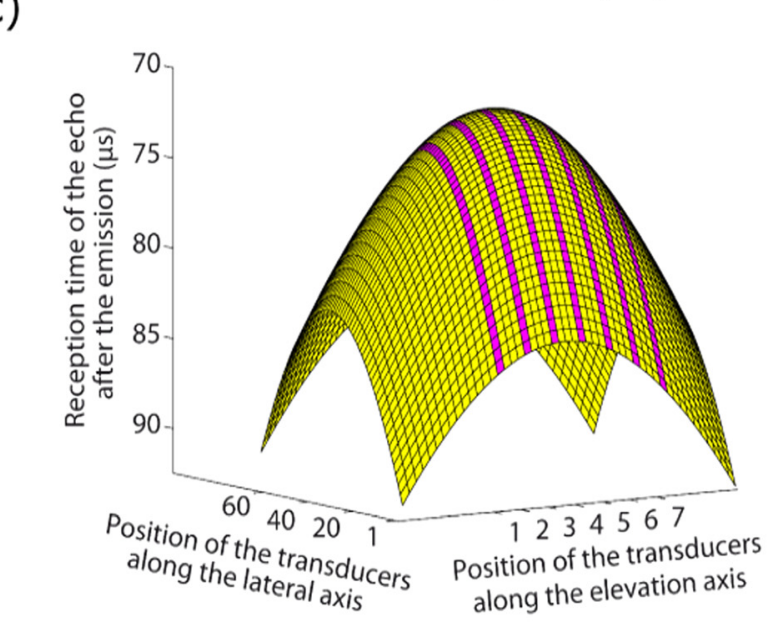

FIG. 2. Single echoes obtained by ultrafast differential imaging can be fitted with a high precision. (a) Distinct events linked to the passage of bubbles within the microchannels are detected by differential imaging. Punctual sources are represented as parabolas in the RF matrix received on the ultrafast scanner. (b) The resulting parabolas are fitted to find their summit and then super-localize the microbubbles. (c) The 3D super-localization is obtained from the fitting of parallel parabolas in the elevation direction.

$\mathrm{n}^{\circ} i$ and $\mathrm{n}^{\circ} i+1$ were subtracted in order to cancel the echoes coming from static structures and isolate the echoes from the bubbles. With an appropriate framerate, the only echoes remaining were those formed by the contrast agents either moving fast or disrupted by one of the emission waves. This very selective high-pass filtering resulted in the isolation of up to 15000 distinct echoes over 50000 images (Figure 2(a)). Given that these echoes were not superimposed, the localization of their respective source was no longer subject to the Abbe limit of resolution. (a)
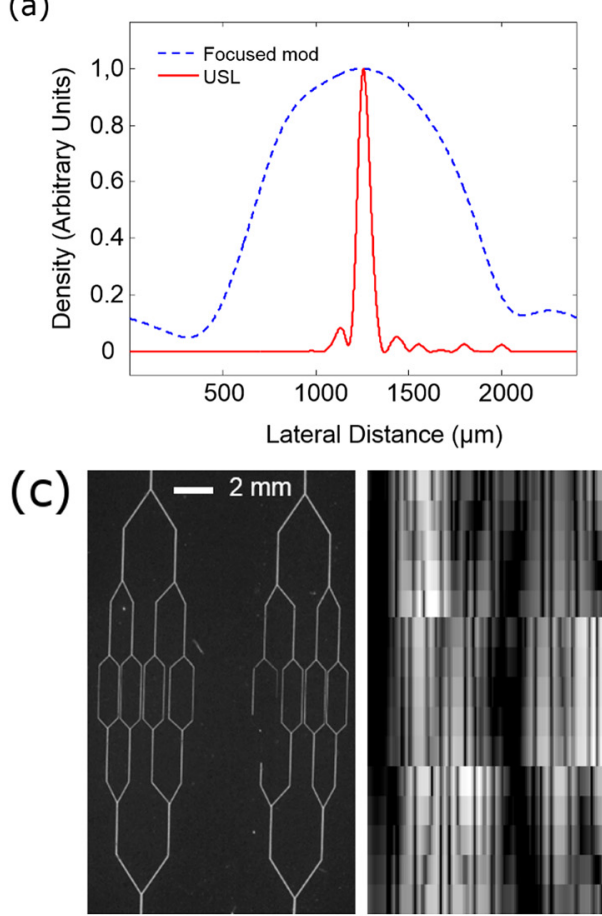

Optical

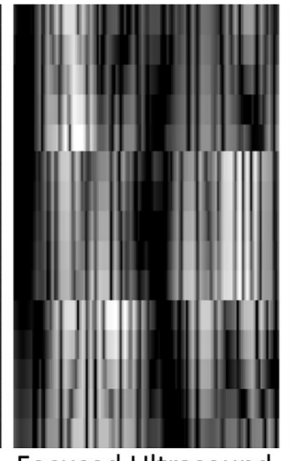

Focused Ultrasound (b)

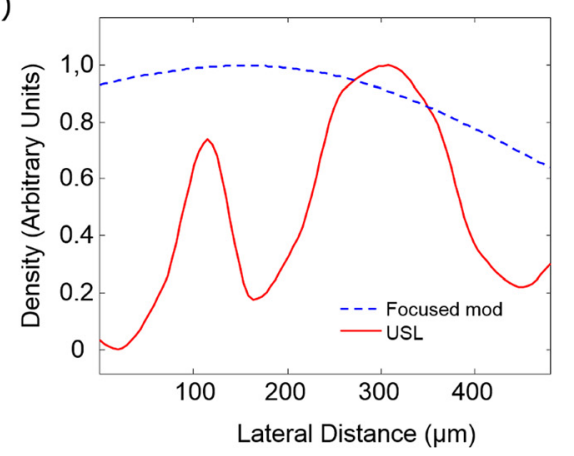

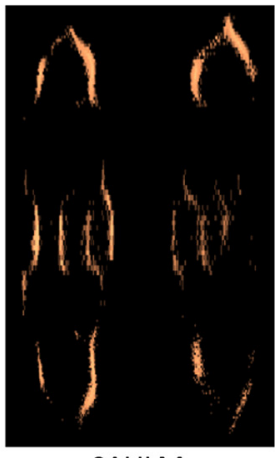

SAULM
SAULM/Optical

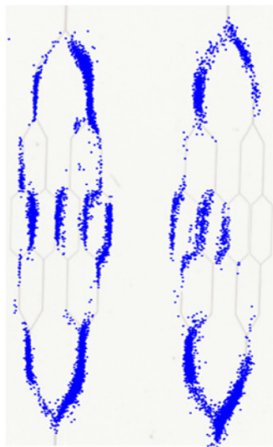

FIG. 3. SAULM grant images with a resolution 13-fold higher than standard echography techniques. (a) The full width at half maximum formed by one channel is $80 \mu \mathrm{m}$ with SAULM whereas it is $1100 \mu \mathrm{m}$ with standard focused imaging. (b) Two channels separated by $200 \mu \mathrm{m}$ are distinguishable with SAULM. (c) Comparison between fluorescence microscopy, standard echography, SAULM, and all the localized sources superimposed with optical image (from left to right); the correlation coefficient between the optical image and the standard ultrasonic image is 0.11 ; the correlation coefficient between the optical image and the SAULM image is 0.22 . 
The localization process consisted in deconvoluting the point spread function (PSF) formed by the microbubble to establish its exact source. When submitted to a plane wave, a small scatterer emits a spherical wave, which is represented by a paraboloid after its detection by a parallel matrix of piezoelectric transducers (Figure 2(c)). The source of the echo can thus be determined by fitting these arrival times with an ultrasonic time-of-flight model assuming the sound speed to be homogeneous in the medium (Figure 2(b)). This fitting process was performed after high-pass filtering, cross-correlation with the emitted plane-wave and automatic exclusion of noise and outliers. A super-localized image consisted in the accumulation of the position of thousands of distinct events.

We defined the accuracy of the imaging system as the full width at half maximum (FWHM) of the intensity of a channel that is much smaller than the wavelength $(\lambda / 11)$. In conventional focused imaging, the profile of the 40 microns bubbles-filled channel had a width of $1100 \mu \mathrm{m}$ (Figure 3(a)). However, when the density of super-localized bubbles was plotted, its width was rather $80 \mu \mathrm{m}$, a 13-fold improvement in accuracy.

Such accuracy was compared to an ad-hoc theoretical localization model and simulations, which both predicted a FWHM of $69 \mu \mathrm{m}$ with the same acoustic parameters. The standard deviations on each of the coordinates were determined from the residual of the fit. The time-of-flight model being non polynomial, we implemented an asymptotic method $^{16}$ to establish the theoretical values (see Eq. (1)). The simulation data were generated by adding Gaussian noise to an exact time-of-flight equation and analyzing the variations in the predicted localization of the source.

$$
\sigma_{x, y, z} \propto \frac{\sigma_{\tau}}{\sqrt{N}},
$$

where $\sigma_{\mathrm{x}, \mathrm{y}, \mathrm{z}}$ is the standard deviation on the resulting position of the source, $\sigma_{\tau}$ is the uncertainty as to the positioning of the maximum of the echo in the time domain and $N$ is the number of transducers used during the acquisition.

The resolution was defined based on the Rayleigh criteria. In conventional focused imaging, two channels separated by $200 \mu \mathrm{m}$ could not be distinguished. However, the profile of the density of super-localized contrast agents demonstrated that these channels, which were distant of $\lambda / 4.5$, could clearly be separated.

Beyond profiles, a full 3D mapping of the microbubble events was derived from the echoes acquired by the transducer matrix (Figure 3(c)). Using conventional focused imaging, the network of channels was impossible to observe since the channels were much smaller and less distant than the wavelength of the acoustic wave. However, when distinct events derived by sono-activated ultrasound localization microscopy were plotted in space, the microfluidic system could be distinguished with an accuracy of $\lambda / 11$. A map of the entire microfluidic system was created (Figure 3(d)), which showed that microbubbles did not flow in all channels equally.

Based on the simple fact that a cloud of randomly distributed microbubbles can act as distinct acoustic sources detected sequentially when imaged at ultrafast frame rates, sono-activated ultrasound localization microscopy can image micro-flow with a resolution and an accuracy up to 13 times the diffraction limit in-vitro. This imaging technique uses a scanner and contrast agents that are both approved clinically. In these conditions, plane-wave imaging was shown to detect very small and rapid changes in populations of microbubbles. ${ }^{11,17}$ In fact, when imaged with a frame rate of $5000 \mathrm{~Hz}$, we observed that echoes from microbubbles flowing through an implanted tumor in a mouse, can be detected individually. Although motion is very limited in-vivo at such frame rates, decorrelation between images due to tissue motion could be corrected with cross-correlation techniques. ${ }^{18}$

Since SAULM uses low frequencies to perform microscopy, it is not limited by the attenuation increase with frequency. However, sono-activation can also be performed at higher frequencies to improve resolutions even more. In the future, SAULM will be implemented in-vivo with shorter wavelength and should lead to the mapping of the entire vascular network of deep seated tissue with a resolution close to the capillary size. This technique breaks the "resolution versus penetration" paradigm of ultrasound imaging and could lead to ultrasound microscopy deep into tissues.

This work was supported by the Agence Nationale de la Recherche (ANR Musli). The authors thank Bracco Research SA for kindly providing the microbubbles.

${ }^{1}$ E. Betzig and J. K. Trautman, Science 257, 189 (1992).

${ }^{2}$ S. T. Hess, T. P. K. Girirajan, and M. D. Mason, Biophys. J. 91, 4258 (2006).

${ }^{3}$ M. J. Rust, M. Bates, and X. Zhuang, Nat. Methods 3, 793 (2006).

${ }^{4}$ M. A. Thompson, M. D. Lew, and W. E. Moermer, Annu. Rev. Biophys. 41, 321 (2012).

${ }^{5}$ S. Cox, E. Rosten, J. Monypenny, T. Jovanovic-Talisman, D. T. Burnette, J. Lippincott-Schwartz, G. E. Jones, and R. Heintzmann, Nat. Methods 9 , 195 (2012).

${ }^{6}$ F. S. Foster, C. J. Pavlin, K. A. Harasiewicz, L. Berube, and A. M. Rauth, IEEE Trans. Ultrason. Ferroelectr. Freq. Control 40, 608 (1993).

${ }^{7}$ K. W. Ferrara, C. R. B. Merritt, P. N. Burns, F. S. Foster, R. F. Mattrey, and S. A. Wickline, Acad. Radiol. 7, 824 (2000).

${ }^{8}$ N. De Jong, P. J. A. Frinking, A. Bouakaz, and F. J. Ten Cate, Ultrasonics 38, 87 (2000).

${ }^{9}$ A. F. H. Lum, M. A. Borden, P. A. Dayton, D. E. Kruse, S. I. Simon, and K. W. Ferrara, J. Controlled Release 111, 128 (2006).

${ }^{10}$ J. Sijl, H. J. Vos, T. Rozendal, N. De Jong, D. Lohse, and M. Versluis, J. Acoust. Soc. Am. 130, 3271 (2011).

${ }^{11}$ O. Couture, S. Bannouf, G. Montaldo, J.-F. Aubry, M. Fink, and M. Tanter, Ultrasound Med. Biol. 35, 1908 (2009).

${ }^{12}$ J. Bercoff, M. Tanter, and M. Fink, IEEE Trans. Ultrason. Ferroelectr. Freq. Control 51, 396 (2004).

${ }^{13}$ M. Fink and M. Tanter, Phys. Today 63(2), 28-33 (2010).

${ }^{14}$ J. Bercoff, G. Montaldo, T. Loupas, D. Savery, F. Mézière, M. Fink, and M. Tanter, IEEE Trans. Ultrason. Ferroelectr. Freq. Control 58, 134 (2011).

${ }^{15}$ E. Mace, G. Montaldo, I. Cohen, M. Baulac, M. Fink, and M. Tanter, Nat. Methods 8(8), 662 (2011).

${ }^{16} \mathrm{~S}$. Huet, A. Bouvier, M.-A. Poursat, and E. Jolivet, Statistical Tools for Nonlinear Regression: A Practical Guide With S-PLUS and R Examples, Springer Series in Statistics (Springer, 2003), p. 31.

${ }^{17} \mathrm{O}$. Couture, M. Fink, and M. Tanter, IEEE Trans. Ultrason. Ferroelectr. Freq. Control 59(12), 2676 (2012).

${ }^{18}$ B. Denarie, T.-A. Tangen, I.-K. Ekroll, N. Rolim, H. Torp, T. Bjastad, and L. Lovstakken, IEEE Trans. Med. Imaging 32, 1265 (2013). 